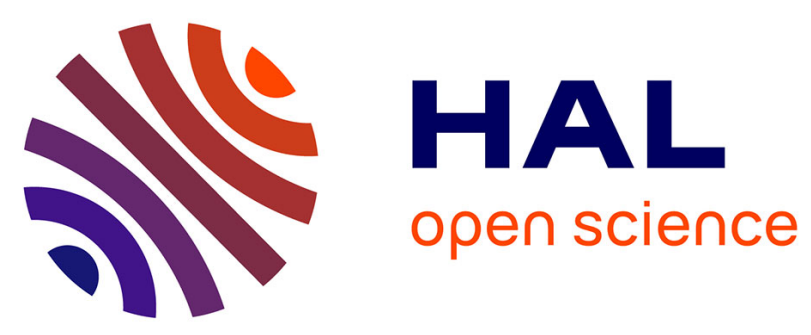

\title{
Monte Carlo Study of sII and sH Argon Hydrates with Multiple Occupancy of Cages
}

\author{
Nikolaos I. Papadimitriou, Ioannis N. Tsimpanogiannis, Aggelos Th. \\ Papaioannou, Athanassios K. Stubos
}

\section{- To cite this version:}

Nikolaos I. Papadimitriou, Ioannis N. Tsimpanogiannis, Aggelos Th. Papaioannou, Athanassios K. Stubos. Monte Carlo Study of sII and sH Argon Hydrates with Multiple Occupancy of Cages. Molecular Simulation, 2008, 34 (10-15), pp.1311-1320. 10.1080/08927020802101734 . hal-00515033

\section{HAL Id: hal-00515033 \\ https://hal.science/hal-00515033}

Submitted on 4 Sep 2010

HAL is a multi-disciplinary open access archive for the deposit and dissemination of scientific research documents, whether they are published or not. The documents may come from teaching and research institutions in France or abroad, or from public or private research centers.
L'archive ouverte pluridisciplinaire HAL, est destinée au dépôt et à la diffusion de documents scientifiques de niveau recherche, publiés ou non, émanant des établissements d'enseignement et de recherche français ou étrangers, des laboratoires publics ou privés. 


\section{Molecular Simulation \\ Journal of \\ Experimental Nanoscience

\section{Monte Carlo Study of sII and sH Argon Hydrates with Multiple Occupancy of Cages}

\begin{tabular}{|c|c|}
\hline Journal: & Molecular Simulation/Journal of Experimental Nanoscience \\
\hline Manuscript ID: & GMOS-2008-0035.R1 \\
\hline Journal: & Molecular Simulation \\
\hline $\begin{array}{r}\text { Date Submitted by the } \\
\text { Author: }\end{array}$ & 24-Mar-2008 \\
\hline Complete List of Authors: & $\begin{array}{l}\text { Papadimitriou, Nikolaos; National Center for Scientific Research } \\
\text { DEMOKRITOS, Environmental Research Laboratory } \\
\text { Tsimpanogiannis, Ioannis; National Center for Scientific Research } \\
\text { DEMOKRITOS, Environmental Research Laboratory } \\
\text { Papaioannou, Aggelos; National Technical University of Athens, } \\
\text { School of Chemical Engineering } \\
\text { Stubos, Athanassios; National Center for Scientific Research } \\
\text { DEMOKRITOS, Environmental Research Laboratory }\end{array}$ \\
\hline Keywords: & argon clathrate hydrates, multiple occupancy, Monte Carlo \\
\hline
\end{tabular}

\section{SCHOLARONE Manuscripts}




\title{
Monte Carlo Study of sII and sH Argon Hydrates with Multiple Occupancy of Cages
}

\author{
N.I. Papadimitriou ${ }^{1,2, *}$, I.N. Tsimpanogiannis ${ }^{1,+}$, A.Th. Papaioannou ${ }^{2}$, A.K. Stubos ${ }^{1}$ \\ ${ }^{1}$ Environmental Research Laboratory, National Center for Scientific Research "Demokritos", \\ Agia Paraskevi 15310, Greece \\ ${ }^{2}$ School of Chemical Engineering, National Technical University of Athens, \\ Zografou 15780, Greece
}

\begin{abstract}
A series of Grand Canonical Monte Carlo simulations are performed to investigate the argon uptake inside sII and $\mathrm{sH}$ argon hydrates at $274 \mathrm{~K}$ and pressures up to $700 \mathrm{MPa}$. Our results indicate that the small cavities of the sII argon hydrate, as well as the small and medium cavities of the sH hydrate are singly occupied without the observation of any multiple occupancy. On the other hand, the large cavities of the sII hydrate are found to be doubly occupied, and the large cavities of the sH hydrate are found to be filled with up to five argon molecules. The results from our simulations are in very good agreement with the available experimental data concerning cage occupancies and with similar results from other types of molecular simulations.
\end{abstract}

Keywords: argon clathrate hydrates; multiple occupancy; Monte Carlo.

\footnotetext{
* Corresponding author: Fax: +302106525004, E-mail: nikpap@ipta.demokritos.gr (N.I. Papadimitriou); tsimpano@usc.edu (I.N. Tsimpanogiannis)

${ }^{+}$Visiting scientist
} 


\section{Introduction}

Gas hydrates are ice-like, crystalline, non-stoichiometric materials that belong to the class of clathrates (i.e. inclusion compounds). They are composed of a framework of hydrogen-bonded water molecules that form cavities (cages) with specific geometry and size, inside which small molecules can be encaged (enclathrated) [1]. The stability of hydrates is due to the interactions (weak, van der Waals-type interactions) between the lattice of water molecules and the enclathrated gas. However, in the absence of the encaged gas, they are not stable.

Three types of hydrates have been identified based on their crystal structure, known as structures sI, sII, and sH. The unit cell of the sI hydrate (cubic Pm3n space group) consists of 46 water molecules that form two types of cavities: the small, a pentagonal dodecahedron $\left(5^{12}\right)$, and the large that is formed by twelve pentagons and two hexagons $\left(5^{12} 6^{2}\right)$. There are two small and six large cavities per unit cell of sI hydrate [2]. The unit cell of the sII hydrate (cubic $F d 3 m$ space group) consists of 136 water molecules that form two types of cavities: the small, a pentagonal dodecahedron $\left(5^{12}\right)$, and the large that is formed by twelve pentagons and four hexagons $\left(5^{12} 6^{4}\right)$. There are sixteen small and eight large cavities per unit cell of sII hydrate [2]. The unit cell of the $\mathrm{sH}$ hydrate (hexagonal $\mathrm{P} 6 \mathrm{mmm}$ space group) consists of 34 water molecules that form three types of cavities: the small, a pentagonal dodecahedron $\left(5^{12}\right)$, the medium that is formed by three tetragons, six pentagons and three hexagons $\left(4^{3} 5^{6} 6^{3}\right)$, and the large that is formed by twelve pentagons and eight hexagons $\left(5^{12} 6^{8}\right)$. There are three small, two medium, and one large cavity per unit cell of sH hydrate [2].

Until recently, it has been believed that small guest molecules (van der Waals diameter less than $5.9 \AA$ ) form sI hydrates, while larger molecules (van der Waals diameter between 5.9 and $6.9 \AA$ ) form sII hydrates. However, recent investigations have shown that some small molecules (e.g. $\mathrm{N}_{2}, \mathrm{O}_{2}, \mathrm{Ar}, \mathrm{Kr}$, and $\mathrm{H}_{2}$ ) form sII hydrates.

Gas hydrates have been investigated for many years because they are involved in a variety of processes [3, 4] of either industrial or scientific/academic interest. Gas hydrates started attracting industrial interest when they were identified [5] as the cause of natural-gas pipelines blocking. This is a major flow-assurance problem and has a significant safety and economical impact. Primary interest in clathrate hydrates arises from their capacity to store large volumes of gas (e.g. if all hydrate cages were filled with a guest molecule, one volume of hydrate would dissociate to approximately 180 volumes (STP) of gas [4]). Large amounts of methane hydrates have been discovered inside oceanic sediments and at permafrost regions, rendering them as possible future energy sources [6]. Hydrates have been considered as an alternative material for storing and/or transporting energy-carrier gases like methane [7] and hydrogen [8]. Storage in the form of hydrate, in oceanic waters, has been proposed as a possible solution to sequestering carbon dioxide [9].

There had been a long-time consensus among researchers that each cage of the hydrate structure can accommodate at most one guest molecule. Furthermore, one of the basic assumptions behind the original statistical thermodynamic theory of van der Waals and Platteeuw (VDWP) [10] and its subsequent modifications [11-14], is that each cavity can contain at most one guest molecule. Recently, however, it has been established that for certain smallsized gas guests (e.g. $\mathrm{N}_{2}, \mathrm{O}_{2}, \mathrm{Ar}$, and $\mathrm{H}_{2}$ ) multiple occupancy of a cage can occur. Note that while over 100 different guest molecules are known to form hydrates [15], very few are known to be capable to accommodate more than one molecules in a single cage. Therefore, there is a need of a generalized theory that can handle multiple filling of cages. Such an effort to 
incorporate multiple cage occupancy was undertaken by Tanaka et al. [16] and Belosludov et al. [17].

If hydrates are to be used as gas-storage materials the number of guest gas molecules inside the cavities is a crucial factor that would determine the total storage capacity of the hydrate (e.g. the storage capacity of hydrogen hydrate is $5.0 \mathrm{wt} . \% \mathrm{H}_{2}$ with quadruple occupancy of the large cavities and double occupancy of the small cavities, while it drops to 3.9 wt. $\% \mathrm{H}_{2}$ with single occupancy of the small cavities while retaining quadruple occupancy of the large cavities). Therefore, if a single cage is occupied by multiple guest molecules, the storage capacity of the hydrate can increase significantly.

The main interest of this work is argon hydrates. Below $460 \mathrm{MPa}$, Ar forms a stable sII hydrate. For the pressure range between 460 and $770 \mathrm{MPa}$ a transformation occurs resulting in a type $\mathrm{sH}$ hydrate, while above $770 \mathrm{MPa}$ a tetragonal structure forms [18]. Note, however, that the pressure boundaries were the "sII-to-sH" structural transformations occur, are still under discussion and different values have been reported [19]. A detailed discussion of this issue is beyond the scope of this study. Inerbaev et al. [20] used first-principle and lattice dynamics calculations to study the electronic, structural, dynamic and thermodynamic properties of Ar hydrate (structures II, H, and tetragonal) considering the effect of multiple-guest molecules in the cages. Manakov et al. [21] studied neutron diffraction patterns of argon hydrates at pressures up to $1000 \mathrm{MPa}$ and reported the filling of the large cavities of $\mathrm{sH}$ structure by five argon atoms. Ogienko et al. [18] reported X-ray diffraction patterns of the sH structure argon hydrate at different temperatures. Their results confirmed the filling of the large cavities of the sH hydrate by five argon atoms. Alavi et al. [22] performed Molecular Dynamics simulations in order to study the stability of type $\mathrm{sH}$ argon hydrate and reported that the most stable argon hydrate has five argon atoms in the large cavities while a single argon atom in the small and medium cavities. Other rare gases (e.g. Kr, Xe) were considered as well and the possibility of multiple occupancies of them was reported. Tanaka et al. [16] extended the VDWP theory to accommodate double occupancy of a single cage. They developed a methodology to calculate the free energy of multiple cage occupancy and predicted that for the case of sII argon hydrate double occupancy dominates over single occupancy of the large cages at pressures above about $300 \mathrm{MPa}$. Itoh et al. [23] conducted Molecular Dynamics calculations in order to investigate the structure and dynamics of doubly occupied sII Ar hydrate. They reported that the total energy and pressure of the system increased significantly when the large cages were doubly occupied.

Additional studies that consider the case of multiple occupancy include, among others, the experimental [24-26] and the theoretical [27-29] works for nitrogen, the experimental [30, 31] and the theoretical [32-37] works for hydrogen, and the theoretical work for carbon dioxide [38].

The main objective of this work is to conduct molecular simulations on sII and $\mathrm{sH}$ argon hydrates in order to calculate cage occupancies as a function of pressure with the Grand Canonical Monte Carlo (GCMC) approach. The simulations are carried out using the software package Accelrys Material Studio ${ }^{\circledR}$. Our results are in good agreement with the experimental and theoretical findings reported earlier. Therefore, we demonstrate the capabilities of the aforementioned software as a first step of an ongoing research on gas storage in hydrates. In the future additional guest gases of significant interest such as nitrogen and hydrogen are going to be considered. 


\section{Simulation details}

\subsection{GCMC approach}

In this work, we have followed the traditional approach of GCMC proposed by Metropolis et al. [39]. A detailed description of this type of simulation is given by Allen and Tildesley [40]. In general, during a Monte Carlo simulation, the ensemble of all possible microstates of a system is sampled in order to calculate average values of the macroscopic properties. By constructing a Markov chain of microstates of the systems, only those microstates with an important contribution to the average properties are sampled. In a GCMC run, this is carried out in the following way [40]: Starting from an initial configuration of the system (initial number and positions of molecules), using a random number generator, a trial "move" is carried out. This "move" could be the creation or destruction of a molecule or its displacement by random distance. In our case, this applies only to the gas molecules while water molecules are kept fixed. The total potential energy of the new configuration is calculated. Based on this energy difference and the gas fugacity, the probability value of the acceptance of the new configuration is calculated using the following Equations:

In case of the creation of a molecule:

$$
\rho_{m \rightarrow n}=\frac{f V}{k T\left(N_{m}-1\right)} \exp \left(-\frac{\Delta U}{k T}\right)
$$

In case of the destruction of a molecule:

$$
\rho_{m \rightarrow n}=\frac{k T N_{m}}{f V} \exp \left(-\frac{\Delta U}{k T}\right)
$$

In case of the displacement of a molecule:

$$
\rho_{m \rightarrow n}=\exp \left(-\frac{\Delta U}{k T}\right)
$$

where, $\rho_{m \rightarrow n}$ is the probability of "moving" from the old configuration $m$ to the new configuration $n, f$ the gas fugacity, $V$ the volume of the simulation box, $k$ is the Boltzmann constant, $T$ the temperature, $N_{m}$ the number of gas molecules in the old configuration and $\Delta U=U_{n}-U_{m}$ is the total potential energy difference between the new and the old configuration.

Then, a random number between 0 and 1 is created and compared with the probability value calculated above. If the random number is lower than the probability, the new configuration is accepted, otherwise it is rejected and the old configuration is the new microstate of the system. In case the probability value is higher or equal to unity, the new configuration is directly accepted. After a large number of such steps, the average properties of the system can be calculated [40]. In a GCMC simulation, the $\mu V T$ statistical ensemble is sampled, which means that the chemical potential $\mu$ (or equally the fugacity $f$ ), the volume $V$ of simulation box and the temperature $T$ are constant during the simulation but the number of gas molecules can vary. 


\subsection{Water and argon models}

Water molecules are simulated using the Extended Simple Point Charge model (SPC/E) [43]. This model was initially fitted using data of several properties of liquid water [43] and it has been shown that it can accurately describe many features of water and its mixtures [22]. Comparatively to other suggested models (e.g. TIP4P, TIP5P), SPC/E was proven to offer sufficient accuracy at a significantly lower computational cost [44]. This model has been previously used for various types of molecular simulations on gas hydrates [22, 27-29, 33-35, 37, $38]$ and it is the most widely used water model for simulations on the specific material. An extended discussion about the efficiency of several water models in predicting hydrate stability is given by Frankcombe and Kroes [45]. The SPC/E model describes van der Waals interactions through a Lennard-Jones potential function and electrostatic interactions through a Coulombic term with a positive charge $\left(q_{H}\right)$ placed on each hydrogen atom and a double negative charge ($2 q_{H}$ ) on the oxygen atom. Values of the parameters for the SPC/E model are given as follows: $\sigma$ $=3.166 \AA, \varepsilon=0.650 \mathrm{~kJ} / \mathrm{mol}$, and $q_{H}=+0.4238$ (electron charge). The $\mathrm{O}-\mathrm{H}$ bond length is taken as $1.00 \AA$. The $\mathrm{H}-\mathrm{O}-\mathrm{H}$ angle varies in the range $105^{\circ}$ and $120^{\circ}$ depending on which type of polygon the water molecule lies on.

Interactions between argon molecules are also described by the Lennard-Jones potential. Electrostatic interactions are ignored for the case of argon due its spherical and non polar nature. There is consensus in the literature $[10,17,46]$ about the Lennard-Jones parameters for argon. This is an additional reason for our selection of argon to study multiple occupancy of cages. The Lennard-Jones parameters for argon proposed by van der Waals and Platteeuw [10] are used in this study $(\sigma=3.408 \AA$ and $\varepsilon=0.9960 \mathrm{~kJ} / \mathrm{mol})$. Parameters for the interaction between argon and water molecules are calculated using the Lorenz-Berthelot combining rules [47]. A cutoff distance of $20 \AA$ was applied (to both physical and electrostatic interactions). 


\subsection{GCMC runs}

Each GCMC run consists of $10^{7}$ steps randomly chosen among creation, destruction and translation with equal probabilities $(33.3 \%)$. The maximum translational displacement is set to 1 $\AA$ which gives a reasonable acceptance ratio [40]. The first 2,500,000 steps of each run are not considered for the calculation of the average properties (equilibration stage). All these simulation parameters are easily defined through the Accelrys Materials Visualizer ${ }^{\circledR}$ interface. Finally, 300,000 configurations are sampled (1 out every 25) and each gas molecule is assigned to its nearest center of cavity. At the end of the simulation, the average occupancy of every cavity is calculated and finally, occupancies are averaged separately for the small and large cavities, as it was observed that no cavity presented a different occupancy trend relatively to the other cavities of the same type. In this way, the occupancy ratio can be calculated, defined as the fraction of cavities occupied by 0-3 gas molecules for sII argon hydrate, and 0-6 gas molecules for sH argon hydrate.

\subsection{Fugacity calculation}

Since simulations are performed at pressures as high as $650 \mathrm{MPa}$, an accurate calculation of the gas fugacity is required. For this reason the Soave-Redlich-Kwong (SRK) Equation of State is used with critical constants for argon taken from [48]. Details about the formulation of the SRK EoS and the fugacity calculation can be found in [47]. To examine the accuracy of this equation, especially at high pressures, the calculated values of the compressibility factor $z$, for argon, are compared with those provided by NIST [49]. As shown in Figure 3 there is very good agreement between the reported and calculated values of compressibility factor $z$. From the same Figure, it becomes obvious that argon presents deviations from the ideal gas behaviour at pressures above $50 \mathrm{MPa}$ (e.g. $z=1.17$ at $50 \mathrm{MPa}$ ).

\section{Results and discussion}

Figure 4 presents the "adsorption isotherms" of argon in type sII and sH hydrates, i.e. the number of argon molecules encaged per unit cell of the hydrate crystal as a function of pressure.

For the case of sII argon hydrate, if all cavities were occupied by one molecule (at most) the isotherm should present a Langmuir trend with a plateau at 24 molecules per unit cell (totally 16 molecules in the small cavities and 8 in the large ones). However, the curve shows a Langmuir-type behaviour only at relatively low pressures (below $100 \mathrm{MPa}$ ). At higher pressures, the argon content exceeds the limit of 24 molecules per unit cell and continues to linearly increase. At the maximum pressure tested $(650 \mathrm{MPa})$, argon content reaches 32 argon molecules per unit cell (34.3 wt. \%). This finding implies that some cavities are occupied by more than one molecule.

Similarly, for the case of type $\mathrm{sH}$ argon hydrate, if all cavities were occupied by one molecule (at most) the isotherm should present a Langmuir trend with a plateau at 6 molecules per unit cell, i.e. 3 molecules in the small cavities, 2 molecules in the medium cavities and 1 in the large ones. At all pressures examined, the argon content exceeds the limit of 6 molecules per unit cell and continues to increase slowly. At the maximum pressure tested $(650 \mathrm{MPa})$, argon content reaches 10 argon molecules per unit cell (34.0 wt. \%). 
For these reasons, the occupancy of each type of cavity has been examined in further detail in order to identify where the guest argon molecules are located.

\section{1 sII argon hydrate}

The results (at $274 \mathrm{~K}$ ) are shown in Figure 5a for the small cavities and Figure $5 \mathrm{~b}$ for the large cavities. The small cavities are occupied by a single molecule with no occurrences of double occupancy observed. On the other hand, almost all the large cavities are filled even at relatively low pressures $(90 \%$ filled at $20 \mathrm{MPa})$. Then, the fraction of singly occupied cavities increases slightly with pressure until it reaches a maximum of $98.3 \%$ at $80 \mathrm{MPa}$. As pressure increases further (above $80 \mathrm{MPa}$ ), doubly occupied large cavities are observed, however, their frequency remains rather low (less than $5 \%$ ) up to approximately $200 \mathrm{MPa}$. At even higher pressures, double occupancy becomes more pronounced and it eventually becomes dominant above $440 \mathrm{MPa}$. At pressures as high as $650 \mathrm{MPa}$ more than $98 \%$ of the large cavities are doubly occupied. No cavities with more than two gas molecules were observed in the entire pressure range considered. Our finding of single occupancy of the small cavities and double occupancy of the large cavities at high pressures is consistent with the theoretical work of Inerbaev et al. [20].

Tanaka et al. [16] reported that when the argon gas is treated as a "realistic" gas, the crossover value where the double occupancy of large cavities becomes dominant over single occupancy is about $300 \mathrm{MPa}$. On the other hand, if the argon gas is treated as an "ideal" gas, the cross-over value is about $1000 \mathrm{MPa}$. Possible explanations for this difference in the cross-over value between our work and Tanaka et al. [16] include the use of different EoS for the description of the argon gas phase and the description of the water-water intermolecular interactions by the TIP4P model in the work of Tanaka et al. [16].

In order to examine the effect of the size of the simulation domain used for the sII hydrate, additional runs were performed where the simulation domain was increased to incorporate eight unit cells ( 2 along each axis). Simulations were conducted for three different pressures $(50,100$, and $200 \mathrm{MPa})$ at $274 \mathrm{~K}$. As shown in Figures 5a, 5b, the results from the eight unit cells (depicted with solid diamonds) are essentially identical with those obtained with one unit cell. Therefore, the use of only one unit cell is well justified since at considerably lower computational cost it yields almost exactly the same results as the simulations with eight unit cells. Obviously, the large number of steps $\left(10^{7}\right)$ during each GCMC run partly compensates for the small size of the simulation box. Recall that for the sII hydrate each unit cell includes a total of 16 small, and 8 large cavities.

The results from the above simulations can be utilized to calculate the Langmuir constants of argon. For the singly occupied small cavities, the dependence of the argon content in the hydrate as a function of the gas fugacity can be described by a simple Langmuir equation, as initially proposed by VDWP [10]:

$$
y_{S}=\frac{C_{S} \cdot f}{1+C_{S} \cdot f}
$$

where:

$y_{S}:$ fraction of singly occupied small cavities

$C_{S}$ : Langmuir constant for singly occupied small cavities $\left(\mathrm{MPa}^{-1}\right)$

$f:$ gas fugacity $(\mathrm{MPa})$ 
For the large cages, a more advanced set of equations that account for double occupancy was proposed by Kuhs et al. [24], and Chazallon and Kuhs [25]:

$$
\begin{gathered}
y_{L 1}=\frac{C_{L 1} \cdot f}{1+C_{L 1} \cdot f+C_{L 1} \cdot C_{L 2} \cdot f^{2}} \\
y_{L 2}=y_{L 1} \cdot C_{L 2} \cdot f
\end{gathered}
$$

where:

$y_{L 1} \quad$ : fraction of singly occupied large cavities

$y_{L 2}$ : fraction of doubly occupied large cavities

$C_{L 1}$ : Langmuir constant for singly occupied large cavities $\left(\mathrm{MPa}^{-1}\right)$

$C_{L 2}$ : Langmuir constant for doubly occupied large cavities $\left(\mathrm{MPa}^{-1}\right)$

The equations above are fitted to the results from the simulations, using the least square procedure and the calculated values of the fitted parameters $C_{S}, C_{L 1}$ and $C_{L 2}$ are as follows: $C_{S}=0.549 \mathrm{MPa}^{-1}, C_{L 1}=1.234 \mathrm{MPa}^{-1}$, and $C_{L 2}=1.129 \times 10^{-4} \mathrm{MPa}^{-1}$. An excellent fitting was achieved for all three types of cavities (small, singly and doubly occupied large cavities), over the entire fugacity range, as shown in Figures 6 and 7.

In energy terms, the isosteric heat of "argon adsorption", which, in fact, represents the energy difference between empty and filled hydrate ranged from 13.3 to $16.7 \mathrm{~kJ} / \mathrm{mol}$. This value corresponds to "physical adsorption" of argon and confirms the rather weak physical interaction between the argon molecules and the lattice of water molecules.

The Accelrys Materials Studio ${ }^{\circledR}$ software enables the visualization of the Monte Carlo simulation results offering a comprehensive insight into the nanostructure of hydrates. For instance, Figure 8 presents the "argon density" profile within the small and large cavities of the hydrate crystal. Red areas represent higher "argon density", i.e. higher probability of finding an argon molecule there. It becomes obvious that argon accumulates in the center of the small cavities. On the other hand, the doubly occupied large cavities do not present the highest argon density at their centers but around a spherical shell with diameter of about $3.2 \AA$. This happens because two argon molecules tend to be placed at symmetric positions relatively to the center.

Figure 9 illustrates the exact positions of the argon molecules in the two types of cavities of the sII argon hydrate. These snapshots are taken from the lowest energy configuration among all configurations sampled during the Monte Carlo simulation.

\section{$3.2 \mathrm{sH}$ argon hydrate}

As mentioned in an earlier section, it has been experimentally shown that approximately above $460 \mathrm{MPa}$ argon forms a type sH hydrate. This type of hydrate consists of cavities with three different sizes: small, medium and large. The large cavities of the sH hydrate are larger relatively to the large cavities of the other hydrate types (sI and sII). Their mean diameter (11.4 $\AA)$ is three times the Van der Waals diameter of argon $(3.8 \AA)$ and consequently one should expect that more than two molecules could be encaged in this cavity. The resulting occupancy obtained from the GCMC simulations of each type of cavity has been examined in detail for the $\mathrm{sH}$ argon hydrate as well. All small and medium cavities were found to be singly occupied. The 
interesting result concerns the large cavities where up to five molecules were found to be encaged. The resulting occupancies as a function of pressure for the large cavities are shown in Figure 10. At $490 \mathrm{MPa}, 85.5 \%$ of the large cavities are occupied by five molecules, $14.3 \%$ by four molecules and only $0.2 \%$ by three. At the maximum pressure of our simulations (650 $\mathrm{MPa})$ the corresponding percentages are: $98 \%$ by five molecules, $2 \%$ by four and no cavity was found to be occupied by three molecules or less. Interestingly, almost no cavities occupied by six or more molecules were observed (their fraction remained lower than $0.02 \%$ even at $650 \mathrm{MPa}$ ). It can be concluded that this cavity cannot host more than five argon molecules because the energy difference induced by the insertion of one additional molecule is too high. This qualitative finding agrees with the results from Molecular Dynamics simulations of Alavi et al. [22]. Our GCMC results regarding the number of the enclathrated argon molecules in each type of cavity are in very good agreement with the available experimental data $[18,21]$. In specific, we estimate that the composition of the $\mathrm{sH}$ hydrate, at $760 \mathrm{MPa}$, is $\mathrm{Ar} \cdot 3.4 \mathrm{H}_{2} \mathrm{O}$ (all large cavities occupied by five Ar molecules) which is exactly the same with the measurements of Manakov et al. [21] while Inerbaev et al. reported a slightly higher Ar content in the hydrate $\left(\mathrm{Ar} \cdot 3.27 \mathrm{H}_{2} \mathrm{O}\right)$. Both these experimental works have confirmed the single occupancy of small and medium cavities of the $\mathrm{sH}$ hydrate by Ar molecules.

Figure 11 illustrates the exact positions of the argon molecules in the medium and large cavities of the $\mathrm{sH}$ argon hydrate (snapshots are taken from the lowest energy configuration among all the configurations sampled during the Monte Carlo simulation). It seems that the five Ar molecules in the large cavity, reside on the corners of a trigonal bipyramid with two of them close to the hexagonal faces of the cavity.

\section{Conclusions}

We have performed a series of Grand Canonical Monte Carlo simulations to investigate the argon uptake inside type sII and $\mathrm{sH}$ argon hydrates. We considered pressures up to $650 \mathrm{MPa}$ and a temperature of $274 \mathrm{~K}$. The simulations were performed using the Accelrys Materials Studio ${ }^{\circledR}$ software package. Our results indicate that the small cavities of the sII argon hydrate, as well as the small and medium cavities of the $\mathrm{sH}$ argon hydrate are singly occupied without the observation of any multiple occupancy. On the other hand, the large cavities of the sII argon hydrate are found to be doubly occupied, and the large cavities of the sH argon hydrate are observed to be filled with up to five argon molecules. The results from our molecular simulations are in very good agreement with previously reported experimental and theoretical works. This agreement was reached using parameters reported in literature without any tuning of their values. The obtained results prove the validity of the methodology and the potential of Accelrys Materials Studio ${ }^{\circledR}$ software to perform simulations for gas storage in such kind of materials. Based on this fact, we are currently investigating other gases of significant interest including hydrogen and nitrogen.

\section{Acknowledgements}

The authors would like to gratefully acknowledge Dr. G.K. Papadopoulos (National Technical University of Athens, School of Chemical Engineering) for providing us with access to the Accelrys Materials Studio ${ }^{\circledR}$ software and for the helpful discussions. 


\section{References}

[1] E.D. Sloan. Clathrate Hydrates of Natural Gases, $2^{\text {nd }}$ ed., Marcel Dekker, New York (1998).

[2] E.D. Sloan. Gas hydrates: Review of physical/chemical properties. Energy \& Fuels, 12, 191 (1998).

[3] C.A. Koh. Towards a fundamental understanding of natural gas hydrates. Chem. Soc. Rev., 31, 157 (2002).

[4] E.D. Sloan. Fundamental principles and applications of natural gas hydrates. Nature, 426, 353 (2003).

[5] E.G. Hammerschmidt. Formation of gas hydrates in natural gas transmission lines. Ind. Eng. Chem., 26, 851 (1934).

[6] K.A. Kvenvolden. A review of the geochemistry of methane in natural gas hydrate. Org. Geochem., 23, 997 (1995).

[7] S. Thomas, R.A. Dawe. Review of ways to transport natural gas energy from countries which do not need the gas for domestic use. Energy, 28, 1461 (2003).

[8] W.L. Mao, H.-K. Mao. Hydrogen storage in molecular compounds. Proc. Natl. Acad. Sci. USA, 101, 708 (2004).

[9] P.G. Brewer, G. Friederich, E.T. Peltzer, F.M. Orr, Jr. Direct experiments on the ocean disposal of fossil fuel CO2. Science, 284, 943 (1999).

[10] J.H. Van der Waals, J.C. Platteeuw. Clathrate solutions. Adv. Chem. Phys., 2, 1 (1959).

[11] W.R. Parrish, J.M. Prausnitz. Dissociation pressures of gas hydrates formed by gas mixtures. Ind. Eng. Chem. Proc. Des. Dev., 11, 26 (1972).

[12] G.D. Holder, G. Corbin, K.D. Papadopoulos. Thermodynamic and molecular properties of gas hydrates from mixtures containing methane, argon, and krypton. Ind. Eng. Chem. Fund., 19, 282 (1980).

[13] V.T. John, G.D. Holder. Choice of cell size in the cell theory of hydrate phase gas-water interactions. J. Phys. Chem., 85, 1811 (1981).

[14] J.B. Klauda, S.I. Sandler. A fugacity model for gas hydrate phase equilibria. Ind. Eng. Chem. Res., 39, 3377 (2000).

[15] P. Englezos. Clathrate hydrates. Ind. Eng. Chem. Res., 32, 1251 (1993).

[16] H. Tanaka, T. Nakatsuka, K. Koga. On the thermodynamic stability of clathrate hydrates IV: Double occupancy of cages. J. Chem. Phys., 121, 5488 (2004).

[17] V.R. Belosludov, O.S. Subbotin, D.S. Krupskii, O.V. Prokuda, R.V. Belosludov, Y. Kawazoe. Microscopic model of clathrate compounds. J. Phys. Conf. Ser., 29, 1 (2006).

[18] A.G. Ogienko, A.V. Kurnosov, A.Y. Manakov, E.G. Larionov, A. I. Ancharov, M.A. Sheromov, A.N. Nesterov. Gas hydrates of argon and methane synthesized at high pressures: Composition, thermal expansion, and self-preservation. J. Phys. Chem. B, 110, 2840 (2006).

[19] H. Hirai, Y. Uchihara, Y. Nishimura, T. Kawamura, Y. Yamamoto, T. Yagi. Structural changes of argon hydrate under high pressures. J. Phys. Chem. B, 106, 11089 (2002).

[20] T.M. Inerbaev, V.R. Belosludov, R.V. Belosludov, M. Sluiter, Y. Kawazoe, J.-I. Kudoh. Theoretical study of clathrate hydrates with multiple occupation. J. Incl. Phenom. Macrocycl. Chem., 48, 55 (2004). 
[21] A.Y. Manakov, V.I. Voronin, A.V. Kurnosov, A.E. Teplykh, V.Y. Komarov, Y.A. Dyadin. Structural investigations of argon hydrates at pressures up to $10 \mathrm{kbar}$. J. Incl. Phenom. Macrocycl. Chem., 48, 11 (2004).

[22] S. Alavi, J.A. Ripmeester, D.D. Klug. Stability of rare gas structure H clathrate hydrates. J. Chem. Phys., 125, 104501 (2006).

[23] H. Itoh, J.S. Tse, K. Kawamura. The structure and dynamics of doubly occupied Ar hydrate. J. Chem. Phys., 115, 9414 (2001).

[24] W.F. Kuhs, B. Chazallon, P.G. Radaelli, F. Pauer. Cage occupancy and compressibility of deuterated $\mathrm{N}_{2}$-clathrate hydrate by neutron diffraction. J. Incl. Phenom. Molec. Recognit. Chem., 29, 65 (1997).

[25] B. Chazallon, W.F. Kuhs. In situ structural properties of $\mathrm{N}_{2^{-}}, \mathrm{O}_{2^{-}}$and air-clathrates by neutron diffraction. J. Chem. Phys., 117, 308 (2002).

[26] S. Sasaki, S. Hori, T. Kume, H. Smimizu. Microscopic observation and in situ Raman scattering studies on high-pressure phase transformations of a synthetic nitrogen hydrate. $J$. Chem. Phys., 118, 7892 (2003).

[27] E.P. Van Klaveren, J.P.J. Michels, J.A. Schouten, D.D. Klug, J.S. Tse. Stability of doubly occupied $\mathrm{N}_{2}$ clathrate hydrates investigated by molecular dynamics simulations. J. Chem. Phys., 114, 5745 (2001).

[28] E.P. Van Klaveren, J.P.J. Michels, J.A. Schouten, D.D. Klug, J.S. Tse. Molecular dynamics simulation study of the properties of doubly occupied $\mathrm{N}_{2}$ clathrate hydrates. J. Chem. Phys., 115, 10500 (2001).

[29] E.P. Van Klaveren, J.P.J. Michels, J.A. Schouten, D.D. Klug, J.S. Tse. Computer simulations of the dynamics of doubly occupied $\mathrm{N}_{2}$ clathrate hydrates. J. Chem. Phys., 117, 6637 (2002).

[30] W.L. Mao, H. Mao, A.F. Goncharov, V.V. Struzhkin, Q. Guo, J. Hu, J. Shu, R.J. Hemley, M. Somayazulu, Y. Zhao. Hydrogen clusters in clathrate hydrates. Science, 297, 2247 (2002).

[31] K.A. Lokshin, Y. Zhao, D. He, W.L. Mao, H.-K. Mao, R.J. Hemley, M.V. Lobanov, M. Greenblatt. Structure and dynamics of hydrogen molecules in the novel clathrate by high pressure neutron diffraction. Phys. Rev. Lett., 93, 125503 (2004).

[32] S. Patchkovskii, J.S. Tse. Thermodynamic stability of hydrogen clathrates. Proc. Natl. Acad. Sci. USA, 100, 14645 (2003).

[33] S. Alavi, J.A. Ripmeester, D.D. Klug. Molecular-dynamics study of structure II hydrogen clathrates. J. Chem. Phys., 123, 024507 (2005).

[34] S. Alavi, J.A. Ripmeester, D.D. Klug. Molecular-dynamics simulations of binary structure II hydrogen and tetrahydrofurane clathrates. J. Chem. Phys., 124, 014704 (2006).

[35] S. Alavi, J.A. Ripmeester, D.D. Klug. Molecular dynamics simulations of binary structure $\mathrm{H}$ hydrogen and methyl-tert-butylether clathrate hydrates. J. Chem. Phys., 124, 204707 (2006).

[36] K. Katsumasa, K. Koga, H. Tanaka. On the thermodynamic stability of hydrogen clathrate hydrates. J. Chem. Phys., 127, 044509 (2007).

[37] N.I. Papadimitriou, I.N. Tsimpanogiannis, A. Th. Papaioannou, A.K. Stubos. Evaluation of the hydrogen storage capacity of pure $\mathrm{H}_{2}$ and binary $\mathrm{H}_{2}$-THF hydrates with Monte Carlo simulations. J. Phys. Chem. C, Submitted (2007).

[38] S. Alavi, T.K. Woo. How much carbon dioxide can be stored in the structure H clathrate hydrates?: A molecular dynamics study. J. Chem. Phys., 126, 044703 (2007). 
[39] N. Metropolis, A.W. Rosenbluth, M.N. Rosenbluth, A.H. Teller, E. Teller. Equation of state calculations by fast computing machines. J. Chem. Phys. 21, 1087 (1953).

[40] M.P. Allen, D.J. Tildesley, Computer Simulation of Liquids; Oxford University Press: New York, USA, 1987.

[41] M. Yousuf, S.B. Qadri, D.L. Knies, K.S. Grabowski, R.B. Coffin, J.W. Pohlman. Novel results on structural investigations of natural minerals of clathrate hydrates. Applied Phys. $A, 78,925$ (2004).

[42] J.D. Bernal, R.H. Fowler. A theory of water and ionic solution, with particular reference to hydrogen and hydroxyl ions. J. Chem. Phys., 7, 515 (1933).

[43] H.J.C. Berendsen, J.R. Grigera, T.P. Straatsma. The missing term in effective pair potentials. J. Phys. Chem., 91, 6269 (1987).

[44] W.L. Jorgensen, J. Chandrasekhar, J.D. Madura, R.W. Impey, M.L. Klein. Comparison of simple potential functions for simulating liquid water. J. Chem. Phys. 79, 926 (1983).

[45] T.J. Frankcombe, G.J. Kroes. Molecular Dynamics Simulations of Type-sII Hydrogen Clathrate Hydrate Close to Equilibrium Conditions. J. Phys. Chem. C, 111, 13044 (2007).

[46] T. Miyoshi, M. Imai, R. Ohmura, K. Yasuoka. Thermodynamic stability of type-I and typeII clathrate hydrates depending on the chemical species of the guest substances. J. Chem. Phys., 126, 234506 (2007).

[47] J.M. Prausnitz, R.N. Lichtenthaler, E.G. Avezedo, Molecular Thermodynamics of FluidPhase Equilibria, Prentice-Hall International Series, New Jersey, USA, (1998).

[48] R.H. Perry, D.W. Green, Perry's Chemical Engineers' Handbook, McGraw-Hill, (1999).

[49] http://webbook.nist.gov 
Figure 1. Crystal structure of a single unit cell of sII hydrate (viewed along one of its edges). The host lattice is shown empty. Red spheres denote the positions of the oxygen atoms of the water molecules and white rods denote the hydrogen atoms of the water molecules.

\section{$82 \times 64 \mathrm{~mm}(200 \times 200$ DPI $)$}


Figure 2. Crystal structure of four unit cells of sH hydrate (viewed along the $\mathrm{z}$ crystallographic axis).

The host lattice is shown empty. Red spheres denote the positions of the oxygen atoms of the water molecules and white rods denote the hydrogen atoms of the water molecules. $82 \times 64 \mathrm{~mm}(200 \times 200$ DPI $)$ 
Page 15 of 23

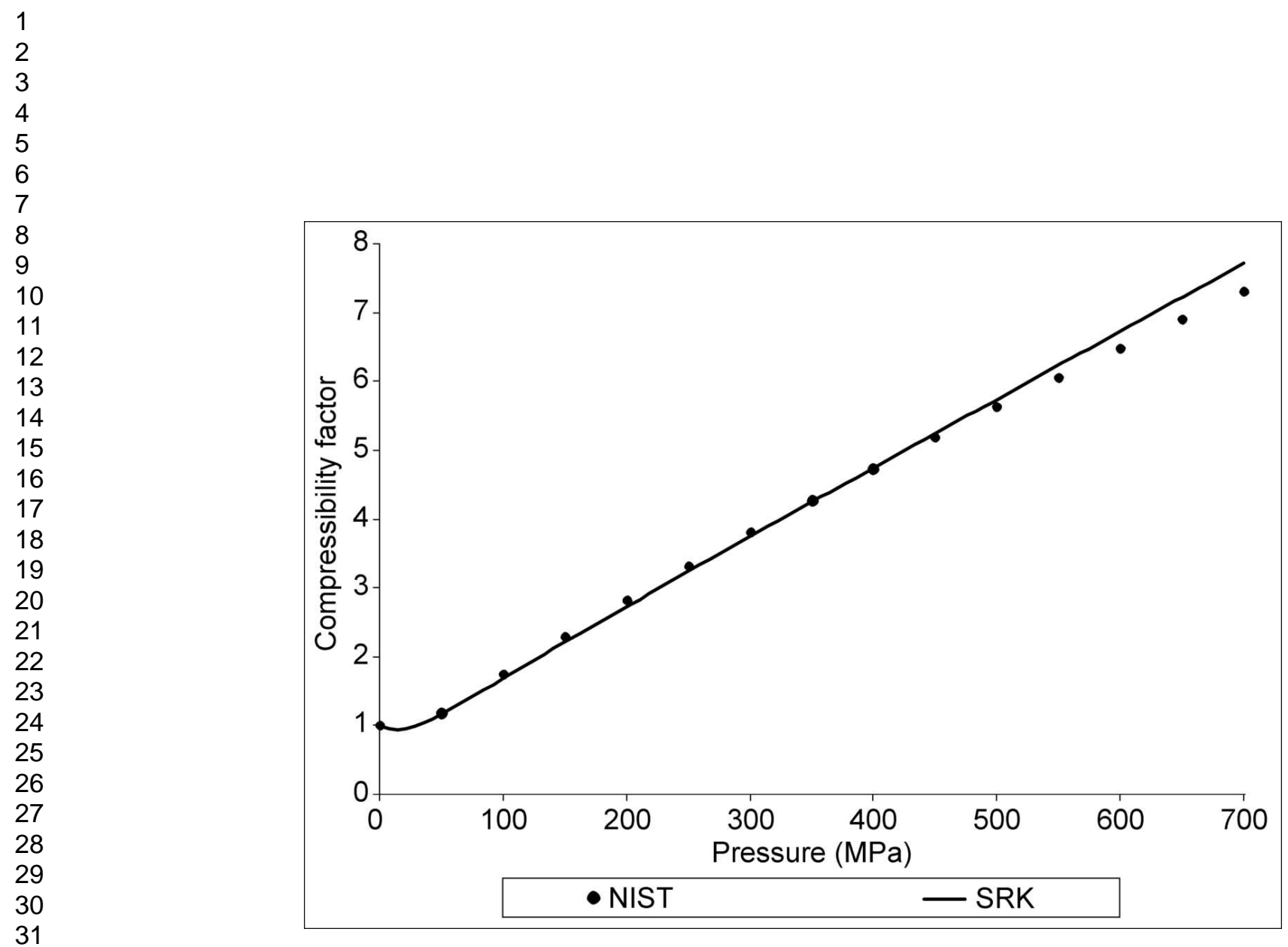

Figure 3. Comparison between the reported (NIST) and calculated (SRK EoS) values for the compressibility factor of argon at $274 \mathrm{~K}$. $59 \times 42 \mathrm{~mm}(600 \times 600 \mathrm{DPI})$

http://mc.manuscriptcentral.com/tandf/jenmol 


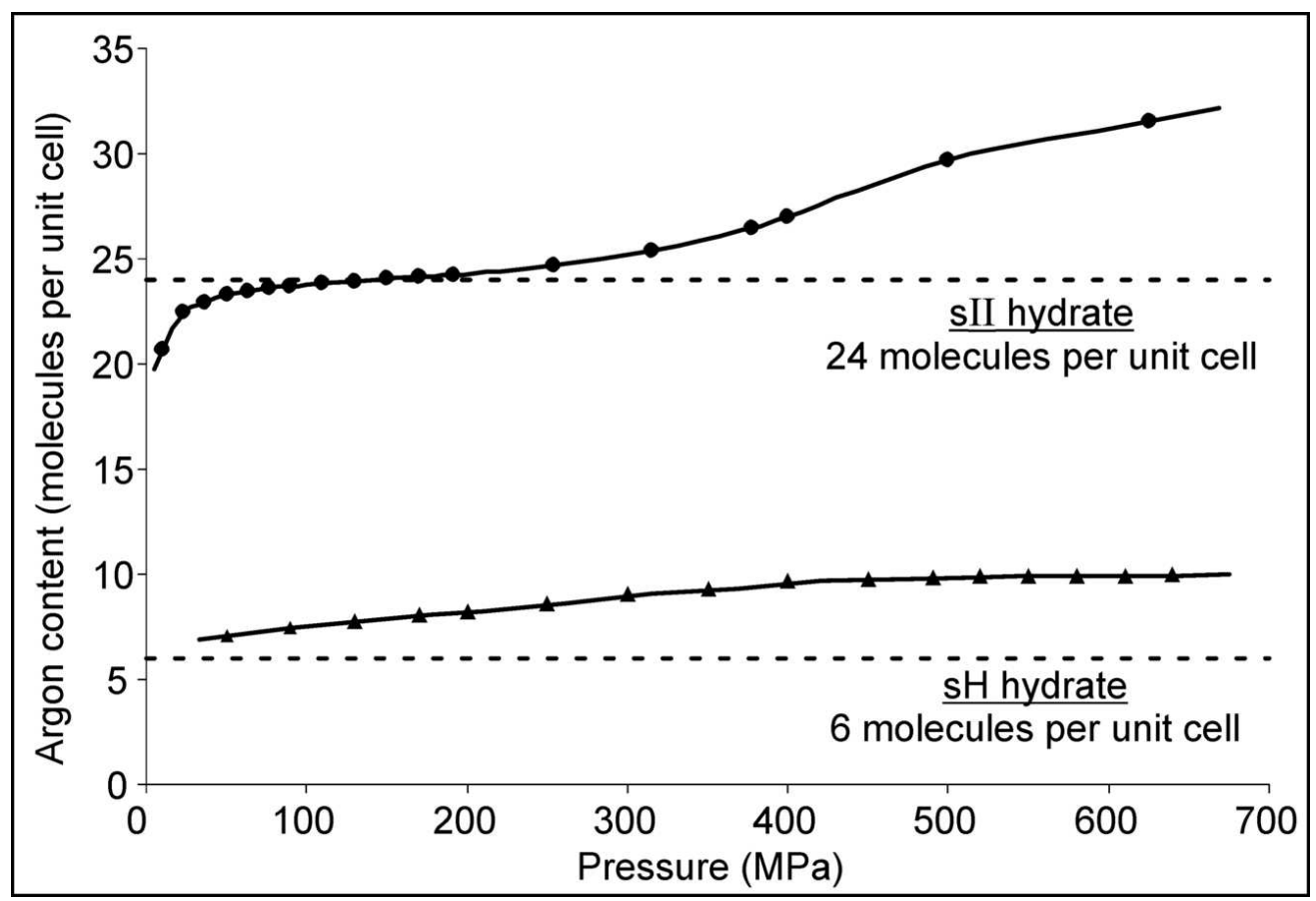

Figure 4. Âl Adsorption isothermsÂn at $274 \mathrm{~K}$ for sII (denoted with circles) and sH (denoted with triangles) hydrates. The dashed horizontal lines denote the special case when all cavities are singly occupied. Solid lines connecting the data points are guides to the eye only. $56 \times 38 \mathrm{~mm}(600 \times 600 \mathrm{DPI})$ 


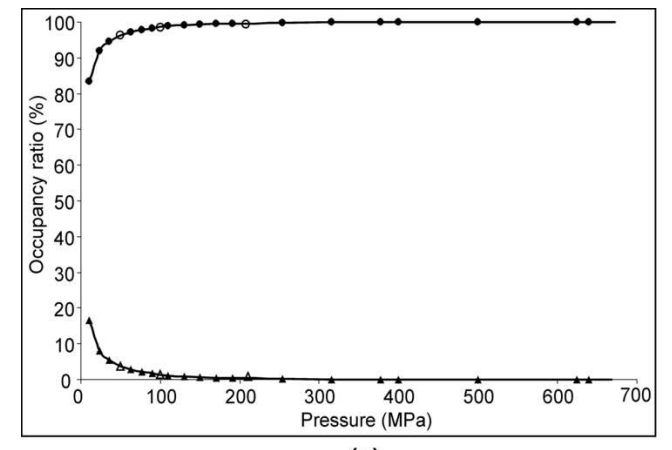

(a)

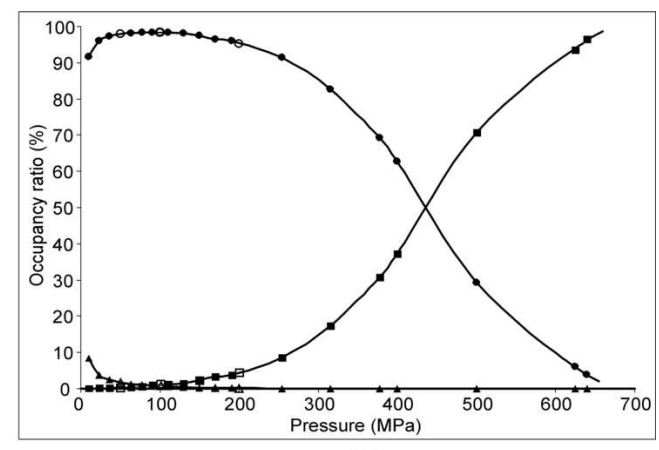

(b)

Figure 5. Occupancy ratio (fraction of cavities occupied by zero (triangles), one (circles) or two (squares) argon molecules) as a function of pressure, at $274 \mathrm{~K}$, for the sII argon hydrate. Results are for (a) small cavities, and (b) large cavities. Empty symbols denote results obtained from eight unit cells, while filled symbols denote results obtained from one unit cell. Solid lines connecting the data points are guides to the eye only.

$63 \times 22 \mathrm{~mm}(600 \times 600 \mathrm{DPI})$ 
Figure 6. Fraction of (singly) occupied small cavities of sII hydrate as a function of pressure, at 274 $\mathrm{K}$. Circles denote the results from the GCMC simulations and solid lines have been derived from fitting to a Langmuir-type equation. $61 \times 46 \mathrm{~mm}(600 \times 600 \mathrm{DPI})$ 


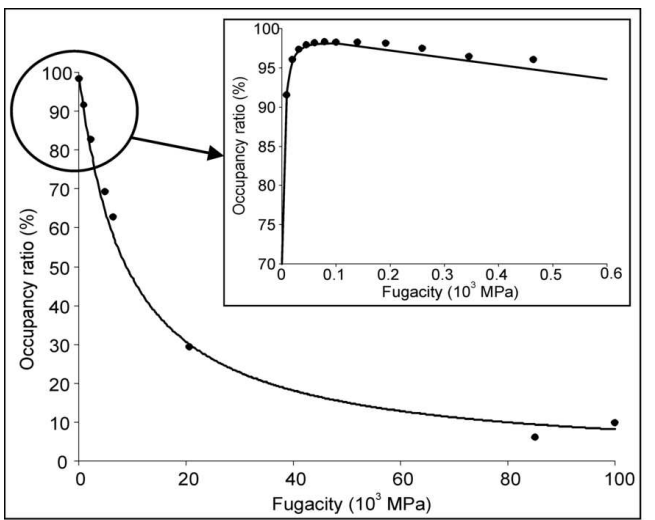

(a)

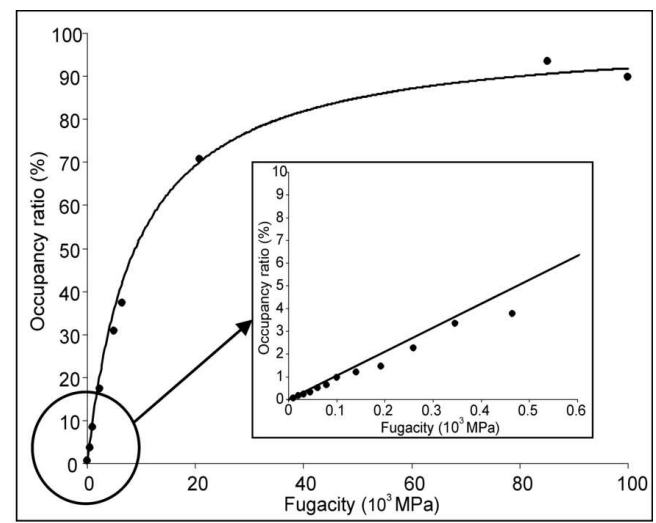

(b)

Figure 7. Fraction of: (a) singly occupied, and (b) doubly occupied large cavities of the sII hydrate as a function of pressure, at $274 \mathrm{~K}$. Circles denote the results from the GCMC simulations and solid lines have been derived from fitting to a two-parameter model $[24,25]$. Embedded plots illustrate the efficiency of the fitting at low pressures. $74 \times 31 \mathrm{~mm}(600 \times 600 \mathrm{DPI})$ 


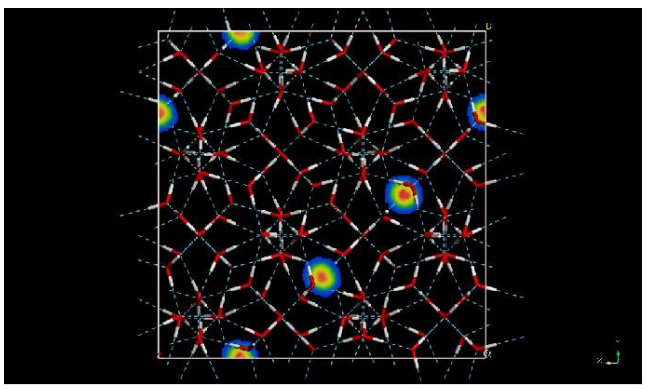

(a)

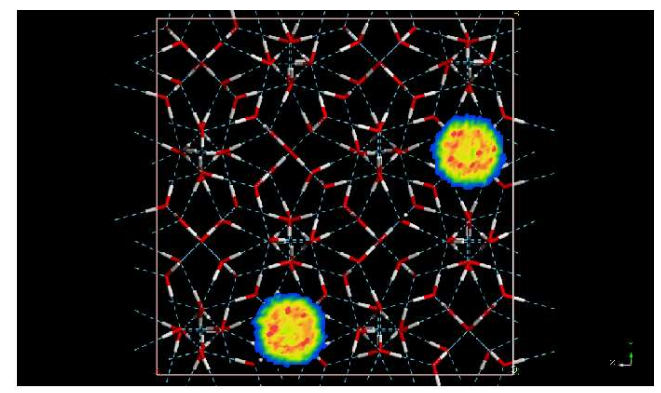

(b)

Figure 8. Visualized results of a GCMC run illustrating Â $\square$ argon densityÂ $\square$ on a cross section of an sII hydrate passing through the centers of (a) the small cavities, and (b) the large cavities. Red areas represent higher densities. $177 \times 57 \mathrm{~mm}(200 \times 200$ DPI $)$ 
Figure 9. Snapshot of the configuration of the argon molecules inside a doubly occupied large cavity (left) and a singly occupied small (right) cavity of sII hydrate. $177 \times 105 \mathrm{~mm}(200 \times 200 \mathrm{DPI})$ 


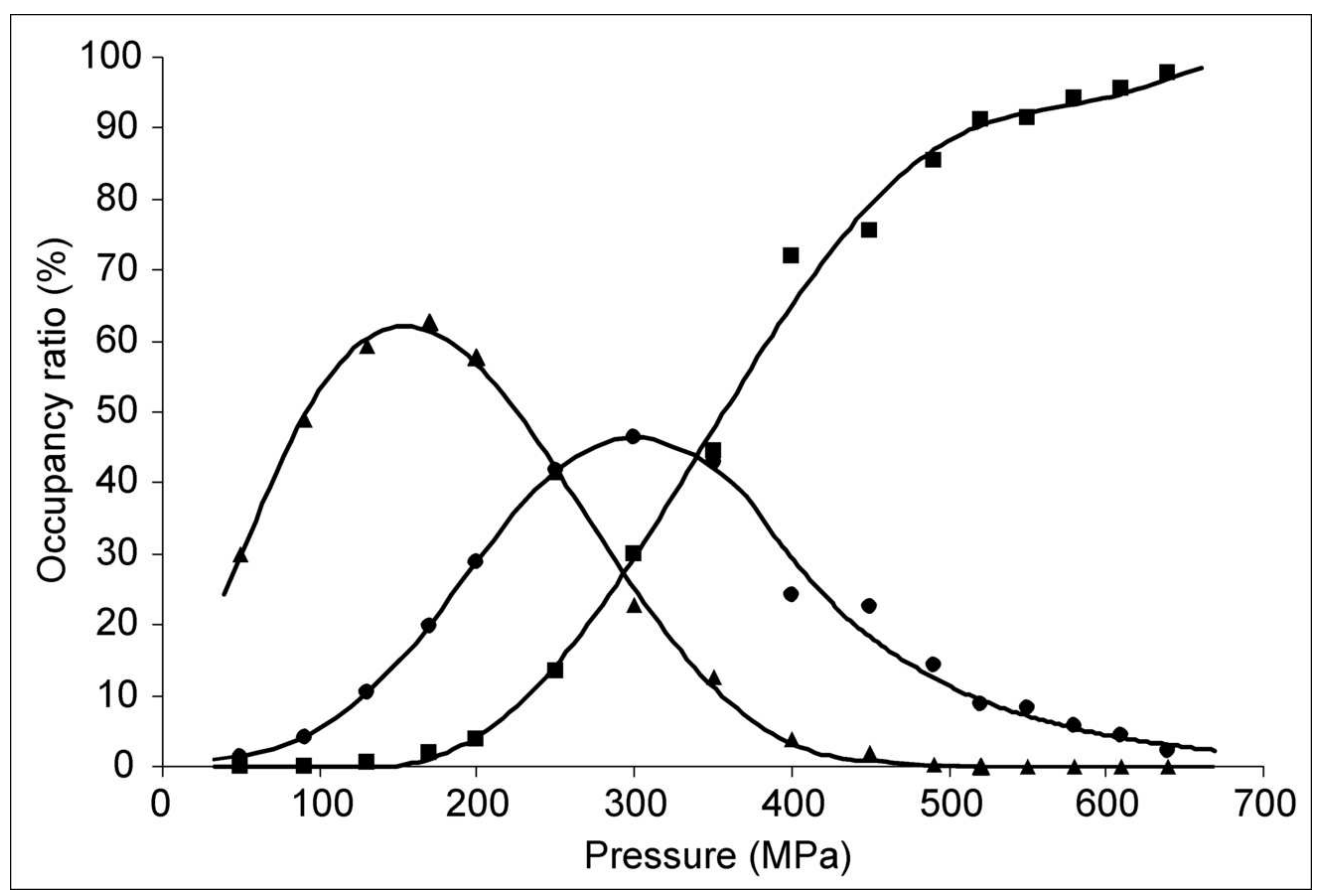

Figure 10. Occupancy ratio of the large cavities as a function of pressure for the $\mathrm{sH}$ argon hydrate, at $274 \mathrm{~K}$ : The data points denote cavities occupied by: three (triangles), four (circles) or five (squares) Ar molecules. Solid lines are guides to the eye only. $55 \times 37 \mathrm{~mm}(600 \times 600$ DPI $)$ 


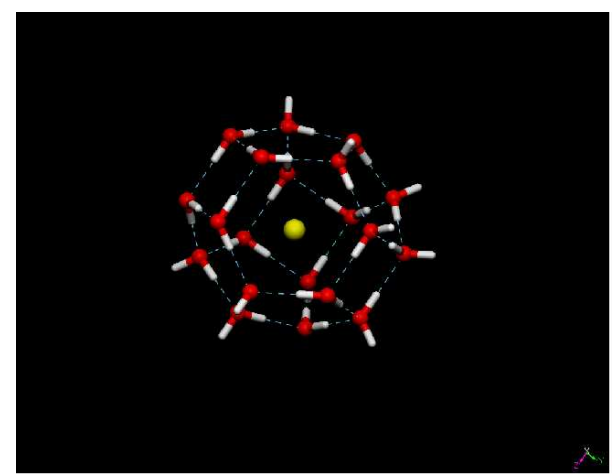

(a)

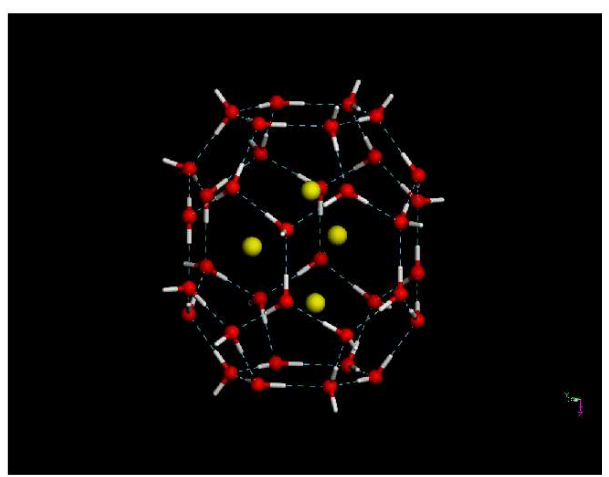

(b)

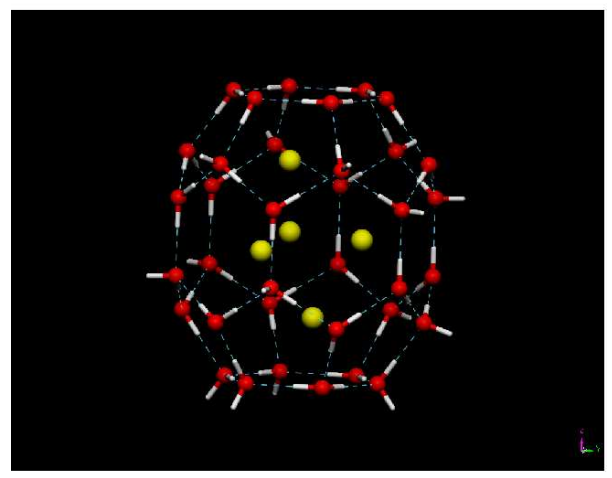

(c)

Figure 11. Snapshots of the configuration of argon molecules inside the cavities of sH hydrate: (a) Medium cavity with single occupancy, (b) large cavity with four argon molecules, and (c) large cavity with five argon molecules. $82 \times 216 \mathrm{~mm}(200 \times 200$ DPI $)$ 